\title{
The Application of Conditional Beta Density Functions to Model Time-Delay Measurement Errors
}

\author{
JOSEPH J. PERRUZZI, MEMBER, IEEE, EDWARD J. HILLIARD, JR., MEMBER, IEEE, AND DAVID V. PEREIRA
}

\author{
(Invited Paper)
}

\begin{abstract}
A conditional beta probability density function is employed to model time-delay measurement errors associated with sensors used in triangulation ranging applications. This type of density function is less restrictive than the beta density used in previous analyses in that end points can be extended out beyond the imposed range constraints and data editing is allowed before range calculations. The resulting conditional range density function is computed along with the mean and variance. Comparisons are made of the pertinent range statistics associated with the beta and conditional beta density functions. Finally, the conditional beta density function range statistics are compared with those obtained from a gated Gaussian density function for equivalent values of time-delay measurement error standard deviation. The Gaussian statisties are determined via Monte-Carlo simulation.
\end{abstract}

\section{INTRODUCTION}

$\mathbf{I}^{\mathrm{N}}$ $\mathrm{N}$ recent years, the triangulation ranging problem has been a topic of considerable research activity [1]-[7]. Triangulation ranging is a method for passively determining the range to an acoustic source from the measured difference in arrival times of a signal wavefront at two receiving sensors. Of particular interest is the effect data measurement errors have on range solution accuracy, and much work has focused on describing measurement statistics [8]-[10]. It is generally assumed that the density function for small time-delay measurement errors (large signal-to-noise ratio-SNR) is Gaussian [4], [10]. However, when the measurement errors become large, the resulting density function is non-Gaussian [4], and a recent paper suggests that it may evolve into a uniform distribution [10]. Since these measurement errors exhibit a mixture of Gaussian (with truncated limits [4]) and uniform characteristics, the beta density function is well suited for modeling purposes because it can easily represent this diverse spectrum of densities. The equation describing such a beta density function will contain four variables, the two end points $\left(A_{1}, A_{2}\right)$ and the two shaping parameters $(a, b)$. Appropriate variation of these parameters leads to accurate approximations of Gaussian-, Rayleigh-, or Maxwell-type distributions. Furthermore, setting the shaping parameters to unity, a uniform density function results. These four variables

Manuscript received March 31, 1986; revised August 6, 1986. This work was sponsored by Naval Underwater Systems Center In-House Independent Exploratory Development under Project B30525.

The authors are with the Naval Underwater Systems Center, Newport, RI 02841-5047.

IEEE Log Number 8714337. determine both the mean and variance, and thus can be related to the magnitude of the measurement errors (SNR).

For small variances, the beta and Gaussian density functions are virtually identical. Increasing the variance causes the beta density function to spread out and finally become uniform with maximum variance. As might be expected, the major advantage of employing a uniform distribution is its inherent mathematical simplicity. Indeed, instead of expressing the pertinent range statistics in terms of hypergeometric series [3], [6], closed form expressions can be obtained [7].

Employing the beta density function leads to analytical expressions for the estimated range density function as well as its mean and variance [3], [5]. Knowledge of the estimated range density function is critical to formulating effective search patterns for acoustic source localization. Contour regions can be generated from these density functions and search operations (e.g., Coast Guard rescue missions for lost vessels) conducted in areas of high probability.

To justify the use of beta distributions for modeling timedelay measurement errors, a comparison must be made with the range statistics obtained when Gaussian distributions are employed to model time-delay measurement errors. Unlike its Gaussian counterpart, the maximum variance of the beta density is finite. In order to compare results for equal measurement error variances, end points of the conventional beta density must be appropriately modified and extended beyond their current limiting values [3], [6]. In particular, a conditional (gated) beta density function must be formulated, which provides for larger values of measurement error variances. Accordingly, the range probability density function is computed, along with the first- and second-order statistics, when time-delay measurement errors are modeled using a conditional beta density function. Comparison of the Gaussian and conditional beta density functions are made for equivalent variances. In addition, resulting range statistics are compared for both the beta and Gaussian density functions. The estimated range statistics for the Gaussian model were generated via computer simulation.

\section{Range Statistics for Generalized Beta Density Time-Delay ERRorS}

For the triangular ranging problem shown in Fig. 1, where one of the bearings $\left(\theta_{2}\right)$ is known exactly and a measurement is 


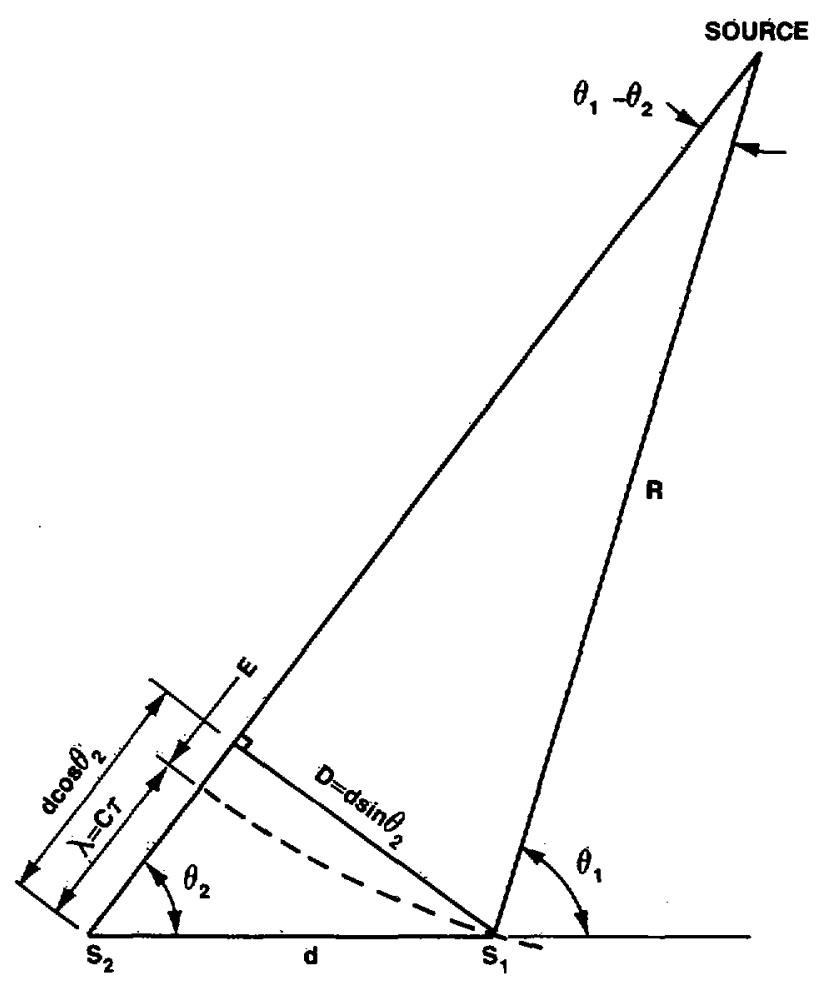

Fig. 1. Geometry of the triangulation ranging problem.

made of the difference in arrival time of an acoustic signal at two spacially separated sensors $\left(S_{1}, S_{2}\right)$, the range is given by [7]

$$
R=\frac{d^{2}+c^{2} \tau^{2}-2 c \tau d \cos \theta_{2}}{2\left(d \cos \theta_{2}-c \tau\right)}
$$

Adding an error $\epsilon$ to the time difference $\tau$, the estimated range is written as

$$
r=\frac{(E-c \epsilon)^{2}+D^{2}}{2(E-c \epsilon)}
$$

where $c$ is the speed of sound in water, $D$ is the effective baseline length, $E / c$ is the maximum allowable time-delay error, and $\epsilon$ is the error in the time-delay measurement. Using a generalized beta density function to model the measurement errors leads to an error density function of the form [7]

$$
f_{\epsilon}(\epsilon)=\frac{\left(\epsilon-A_{2}\right)^{a-1}\left(A_{1}-\epsilon\right)^{b-1}}{\left(A_{1}-A_{2}\right)^{a+b-1} B(a, b)}
$$

where $A_{\mathrm{I}}, A_{2}$ are the maximum and minimum limits on the time-delay errors, $a, b$ are the positive shaping parameters, and $B(a, b)$ is the beta function. The mean and standard deviation of $f_{\epsilon}(\epsilon)$ are given by

$$
m_{\epsilon}=\frac{a A_{1}+b A_{2}}{a+b}
$$

and

$$
\sigma_{\epsilon}=\frac{\sqrt{a b}\left(A_{1}-A_{2}\right)}{(a+b) \sqrt{a+b+1}} .
$$

SHAPING PARAMETERS $\mathrm{a}=\mathrm{b}=5$

\begin{tabular}{|c|c|c|c|c|}
\hline CASE & $\begin{array}{c}\epsilon_{1} \\
(\mathrm{~ms})\end{array}$ & $\begin{array}{c}\epsilon_{2} \\
(\mathrm{~ms})\end{array}$ & $\begin{array}{c}\text { MEAN } \\
(\mathrm{ms})\end{array}$ & $\begin{array}{c}6 \\
(\mathrm{~ms})\end{array}$ \\
\hline 1 & .0227 & $A_{2}$ & -.00267 & .0217 \\
2 & .0227 & -.027 & -.0016 & .01177 \\
3 & $A_{1}$ & $A_{2}$ & 0 & .015 \\
\hline
\end{tabular}

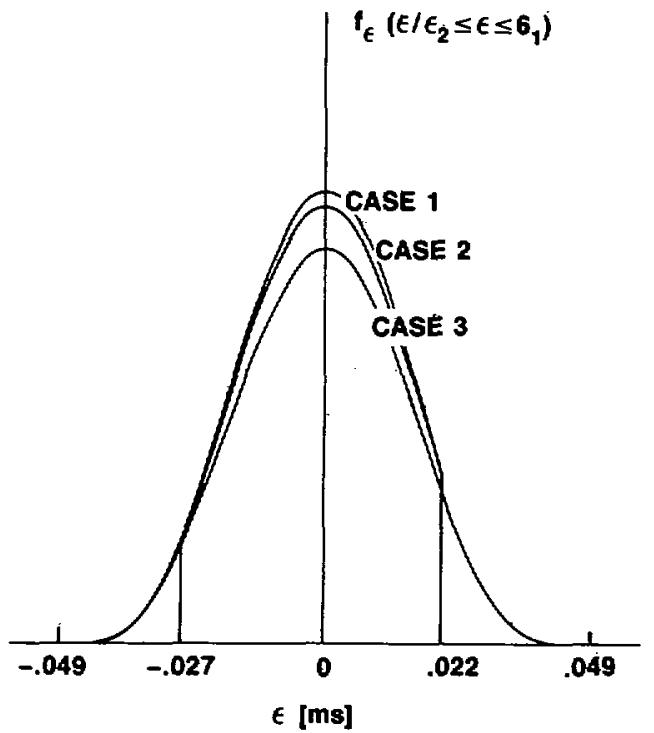

Fig. 2. Conditional beta density function to model time-delay measurement errors.

\section{Condittonal Beta Density Function}

The physical end points of the beta density function given in (3) can be extended, but in order to generate range estimates which remain finite, the density function must be truncated which results in a conditional density function for modeling the measurement errors. If $\epsilon_{1}$ and $\epsilon_{2}$ are the truncated maximum and minimum limits on the time-delay measurement errors, then from [11], a conditional beta density function can be written as

$$
f_{\epsilon}\left(\epsilon \mid \epsilon_{2} \leq \epsilon \leq \epsilon_{1}\right)=\frac{f_{\epsilon}(\epsilon)}{\int_{\epsilon_{1}}^{\epsilon_{2}} f_{\epsilon}(\epsilon) d \epsilon} .
$$

Evaluating (6), the resulting conditional beta density function becomes

$$
f_{\epsilon}\left(\epsilon \mid \epsilon_{2} \leq \epsilon \leq \epsilon_{1}\right)=\frac{\left(\epsilon-A_{2}\right)^{a-1}\left(A_{1}-\epsilon\right)^{b-1}}{\left(A_{1}-A_{2}\right)^{a+b-1}\left[I_{x 1}(a, b)-I_{x 2}(a, b)\right]}
$$

where

$$
\begin{aligned}
& x_{1}=\frac{\epsilon_{1}-A_{2}}{A_{1}-A_{2}} \\
& x_{2}=\frac{\epsilon_{2}-A_{2}}{A_{1}-A_{2}}
\end{aligned}
$$

and $I_{x}(a, b)$ is the incomplete beta function [12]. Comparing 


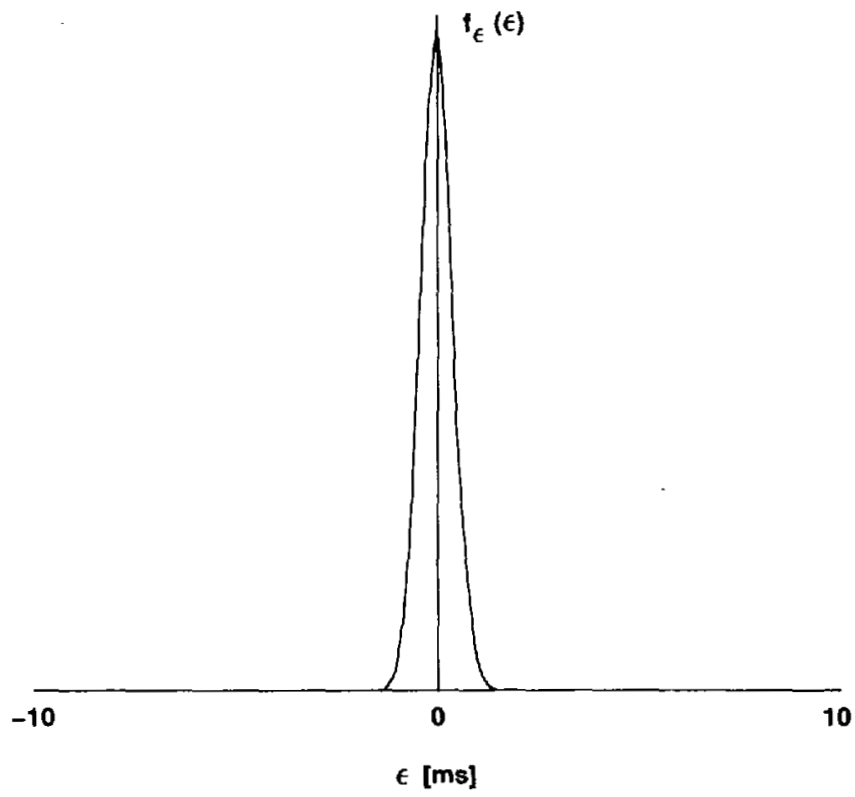

(a)

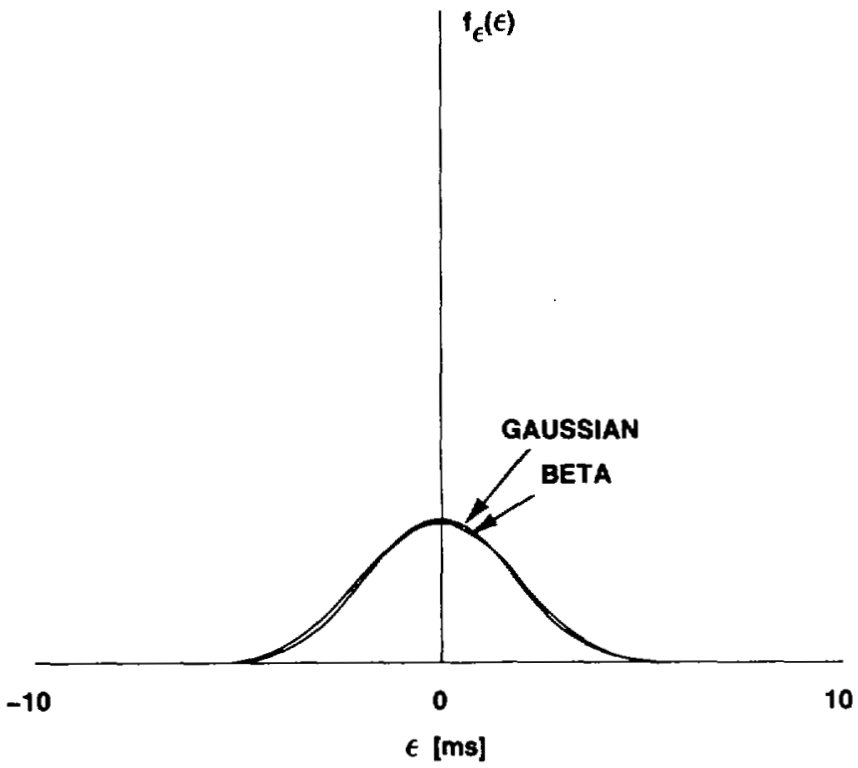

(c)

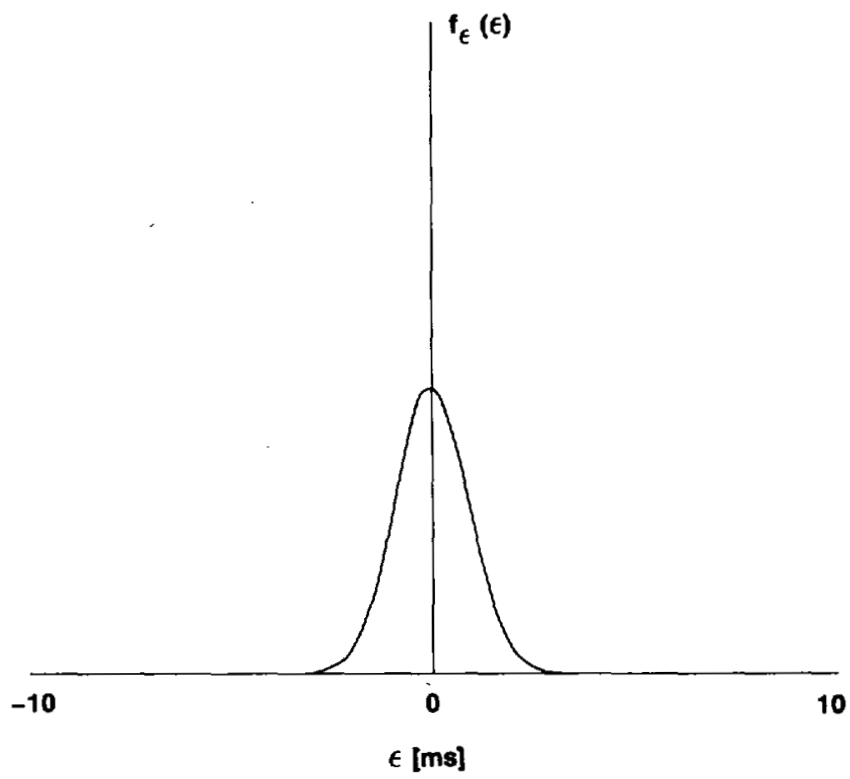

(b)

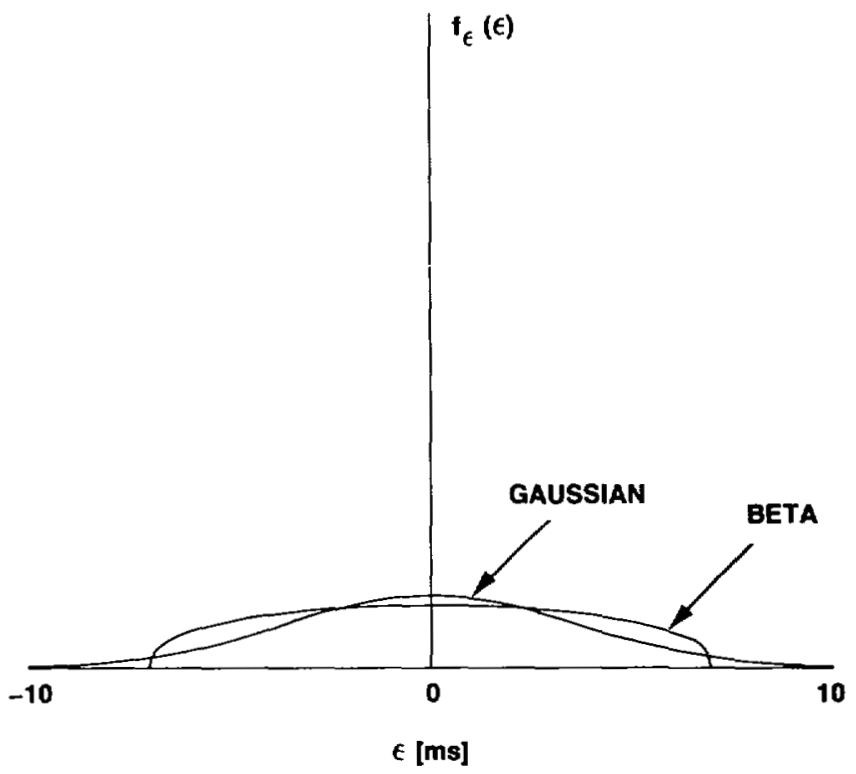

(d)

Fig. 3. (a) Beta and Gaussian density function for $\sigma=0.4$. (b) Beta and Gaussian density function for $\sigma=0.9$. (c) Beta and Gaussian density functions for $\sigma=1.8$. (d) Beta and Gaussian density functions for $\sigma=3.6$.

(3) and (7), it is apparent that the only differences between the beta density and the conditional beta density functions are the incomplete beta functions, $I_{x 1}(a, b)$ and $I_{x 2}(a, b)$, which scale the conditional beta density function. The scale factor increases the height of the conditional density function to account for the loss of area from truncating the original density at $\epsilon_{1}$ and $\epsilon_{2}$ as can be seen from Fig. 2.

Through proper selection of shaping parameters and end points, the beta density function can be employed to model or approximate a wide variety of density functions (uniform, ramp, Gaussian, Rayleigh, etc.). Fig. 3(a)-(d) contains arbitrary plots of beta and Gaussian density functions for equivalent standard deviations $\sigma$. For the beta density, the end points were fixed at a constant value, and the shaping parameters varied to change $\sigma$ in all the figures. For small values of $\sigma$, the two density functions are identical (see Fig. 3(a) and (b)). As $\sigma$ increases, the differences between the two densities become apparent. This results from using fixed end points for the beta density. However, these end points could have been extended farther out to represent larger bounding errors, and other values for the shaping parameters subsequently found to keep $\sigma$ the same thus resulting in a better match between the beta and Gaussian density functions contained in Fig. 3(c) and (d).

Due to the infinite tails of the Gaussian density function, truncation is essential to achieve finite range estimates [4]. Fig. 4(a)-(d), depicts conditional beta and Gaussian density functions which were truncated at arbitrary points. Once 


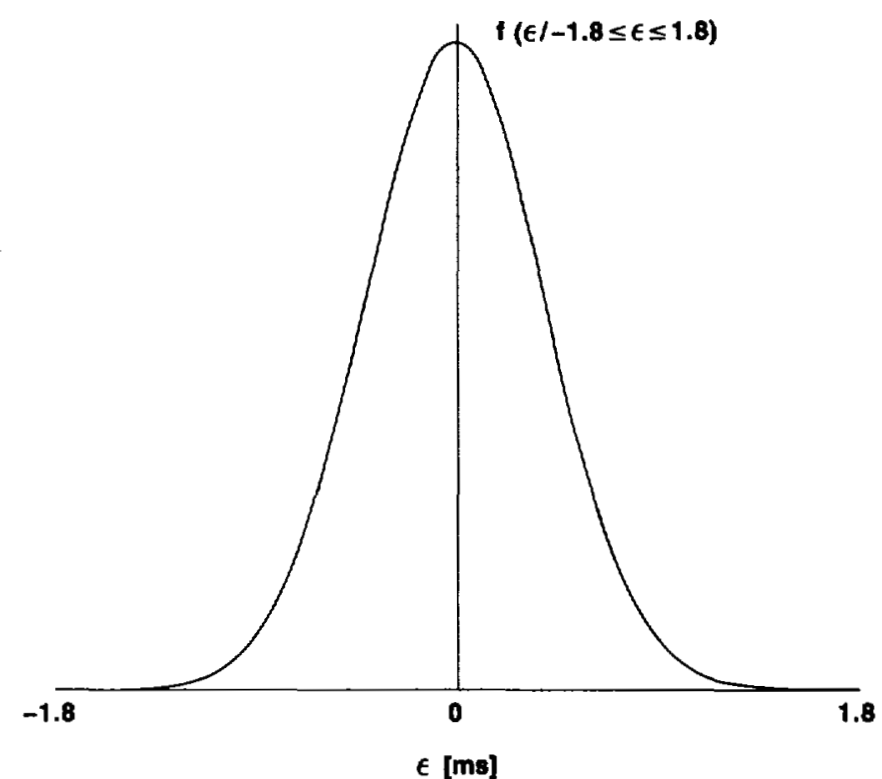

(a)

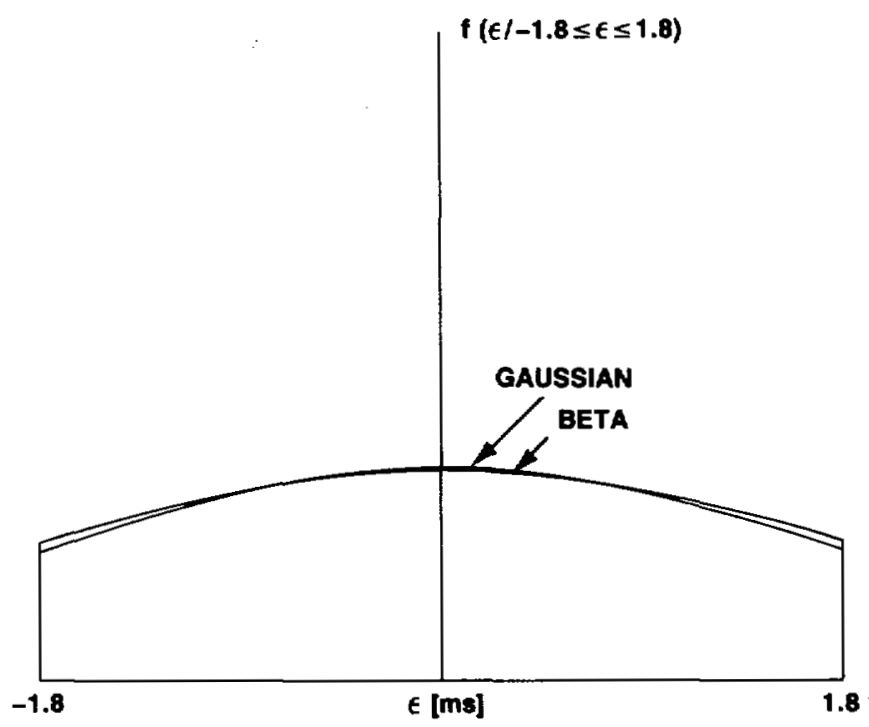

(c)

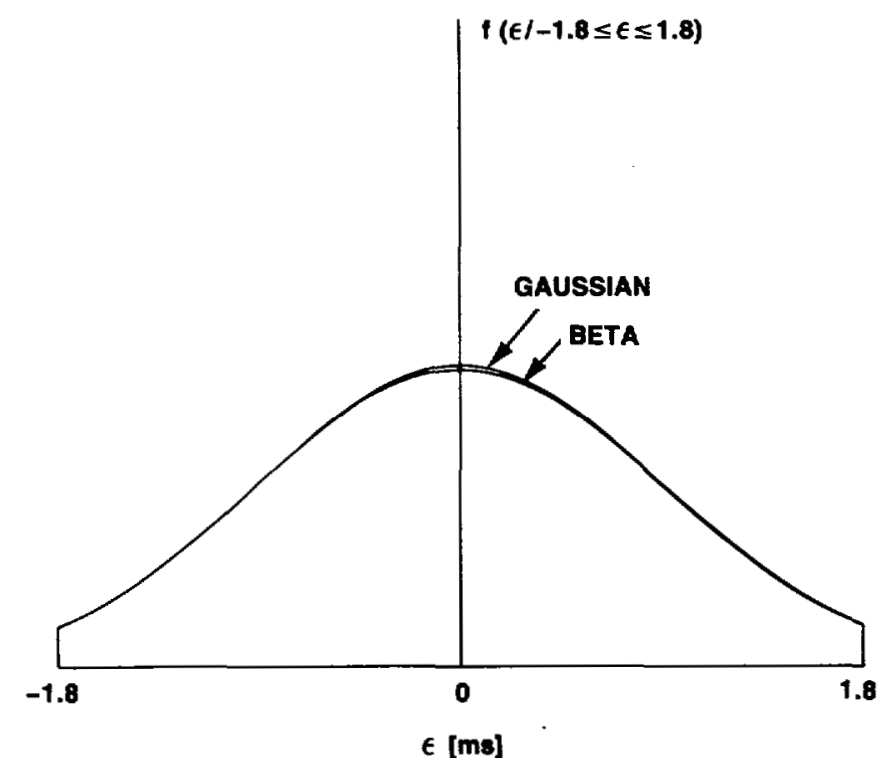

(b)

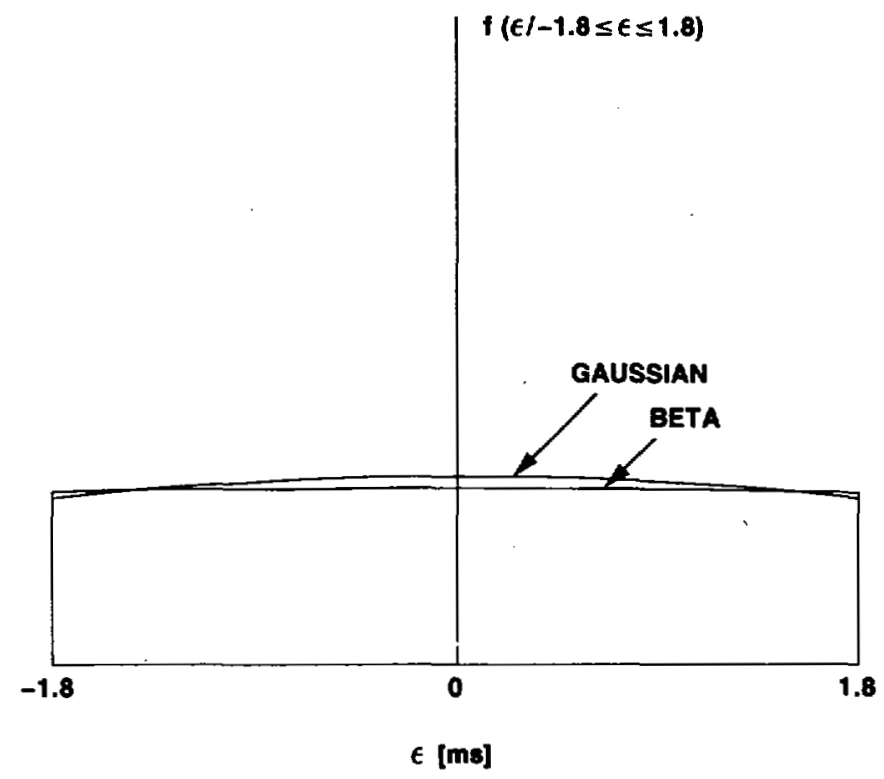

(d)

Fig. 4. (a) Truncated beta and Gaussian density functions for $\sigma=0.4$. (b) Truncated beta and Gaussian density functions for $\sigma=0.9$. (c) Truncated beta and Gaussian density functions for $\sigma=1.8$. (d) Truncated beta and Gaussian density functions for $\sigma=3.6$.

again, for small $\sigma$, there is little difference between the two densities. Even when $\sigma$ increases, the two densities shown in range in (2) is also a conditional density function. The probability density function for $r$, then takes the form [6]

$$
f_{r}\left(r \mid r_{\min } \leq r \leq r_{\max }\right)=\frac{r-\sqrt{r^{2}-D^{2}}\left[\left(E-c A_{2}\right)-\left(r-\sqrt{r^{2}-D^{2}}\right)\right]^{a-1}\left[\left(c A_{1}-E\right)+\left(r-\sqrt{r^{2}-D^{2}}\right)\right]^{b-1}}{\sqrt{r^{2}-D^{2}}\left[c\left(A_{1}-A_{2}\right)\right]^{a+b-1} B(a, b)\left[I_{x 1}(a, b)-I_{x 2}(a, b)\right]}
$$

Fig. 4(c) and (d) are in close agreement. As was noted in [10], large measurement errors are uniformly distributed. Both conditional density functions follow this trend as shown in Fig. 4(c) and (d).

\section{Condrtional Range Density Function}

When the error associated with the time-delay measurement is given by (7), the resultant density function for the estimated where

$$
\begin{gathered}
r_{\min }=\frac{\left(E-c \epsilon_{2}\right)^{2}+D^{2}}{2\left(E-c \epsilon_{2}\right)} \\
r_{\max }=\frac{\left(E-c \epsilon_{1}\right)^{2}+D^{2}}{2\left(E-c \epsilon_{1}\right)} .
\end{gathered}
$$


From (11) and (12) it is seen that the limits imposed upon the time-delay errors $\left(\epsilon_{1}\right.$ and $\left.\epsilon_{2}\right)$ bound the limits on the range calculations.

The Mellin transform [14] is employed to determine the moments for the estimated range, and the results are

$$
\begin{gathered}
F_{m}(2)=\frac{D^{2}\left(x_{1}^{a}-x_{2}^{a}\right)}{2\left(E-c A_{2}\right) I \phi} \sum_{k=0}^{\infty}\left(\bar{x}_{1}^{k}-\bar{x}_{2}^{k}\right) \\
\cdot \sum_{n=0}^{b-1} \frac{(1-b, n)}{(a+n+k) n !}\left(x_{1}^{n}-x_{2}^{n}\right) \\
+\frac{E-c A_{2}}{2}\left[1-\frac{a I 1}{(a+b) I \phi} y\right] \\
I 1=I_{x 1}(a+1, b)-I_{x 2}(a+1, b) \\
\bar{x}_{1}=\frac{c\left(\epsilon_{1}-A_{2}\right)}{E-c A_{2}} \\
\bar{x}_{2}=\frac{c\left(\epsilon_{2}-A_{2}\right)}{E-c A_{2}} \\
y=\frac{c\left(A_{1}-A_{2}\right)}{E-c A_{2}} .
\end{gathered}
$$

The second moment is given by

$$
\begin{aligned}
\mathcal{F}_{m}(3)= & \frac{D^{4}\left(x_{1}^{a}-x_{2}^{a}\right)}{4\left(E-c A_{2}\right)^{2} I \phi} \sum_{k=0}^{\infty}\left(\bar{x}_{1}^{k}-\bar{x}_{2}^{k}\right)(k+1) \\
& \cdot \sum_{n=0}^{b-1} \frac{(1-b, n)}{(a+n+k) n !}\left(x_{1}^{n}-x_{2}^{n}\right) \\
& +\frac{D^{2}}{2}+\frac{\left(E-c A_{2}\right)^{2}}{4}\left[1-\frac{2 a I 1}{(a+b) I \phi} y\right. \\
& \left.+\frac{a(a+1) I 2}{(a+b)(a+b+1) I \phi} y^{2}\right] \\
I 2= & I_{x 1}(a+2, b)-I_{x 2}(a+2, b) .
\end{aligned}
$$

Using the time-delay density functions from Fig. 2 for $a=b$ $=5$, the corresponding range density functions are shown in Figs. 5, 6, and 7 for no gates, 1 gate, and 2 gates, respectively. The minimum and maximum values for the range density function $f_{r}(r)$, in Fig. 5 are found from the bounds on the time-delay density function $f_{\epsilon}(\epsilon)$ (case 3 ). Notice that the mean value for $f_{\epsilon}(\mathrm{\epsilon})$ is zero, but the estimated range exhibits a positive bias. Fig. 6 shows a plot of the conditional range density function $f_{r}\left(r \mid r \leq r_{\max }\right)$ when $f_{\epsilon}\left(\epsilon \mid \epsilon \leq \epsilon_{1}\right)$ is used to model the time-delay errors (case 1 in Fig. 2). Only the upper limit was gated which reduced the mean value for the estimated range. When the density function is gated on both sides (case 2 in Fig. 2), the mean range value now increases but the variance decreases as shown in Fig. 7. (Note: $1 \mathrm{kyd}=$ $0.9144 \mathrm{~km}$.)
TRUE RANGE $=\mathbf{4 0} \mathrm{KYD}$

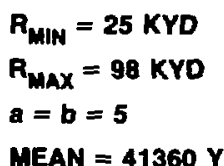

$6_{r}=8000 \mathrm{YD}$

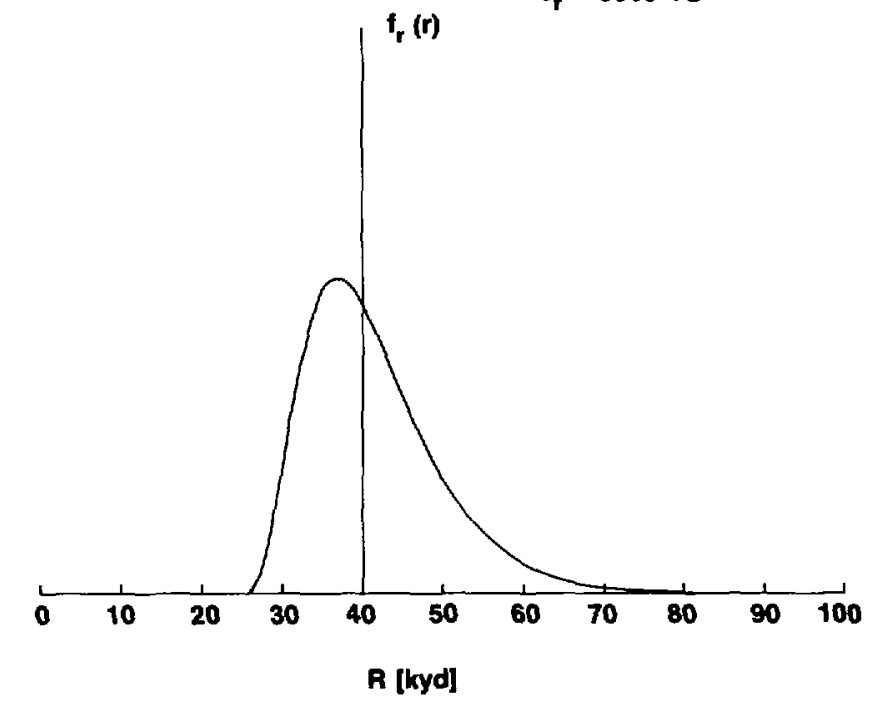

Fig. 5. Range density function.

TRUE RANGE $=\mathbf{4 0} \mathrm{KYD}$

$$
\begin{aligned}
& R_{\text {miY }}=25 \mathrm{KYD} \\
& R_{\text {MAX }}=55 \mathrm{KYD} \\
& a=b=5
\end{aligned}
$$$$
\text { MEAN }=40055 \text { YD }
$$

STANDARD DEVIATION $=6259$ YD

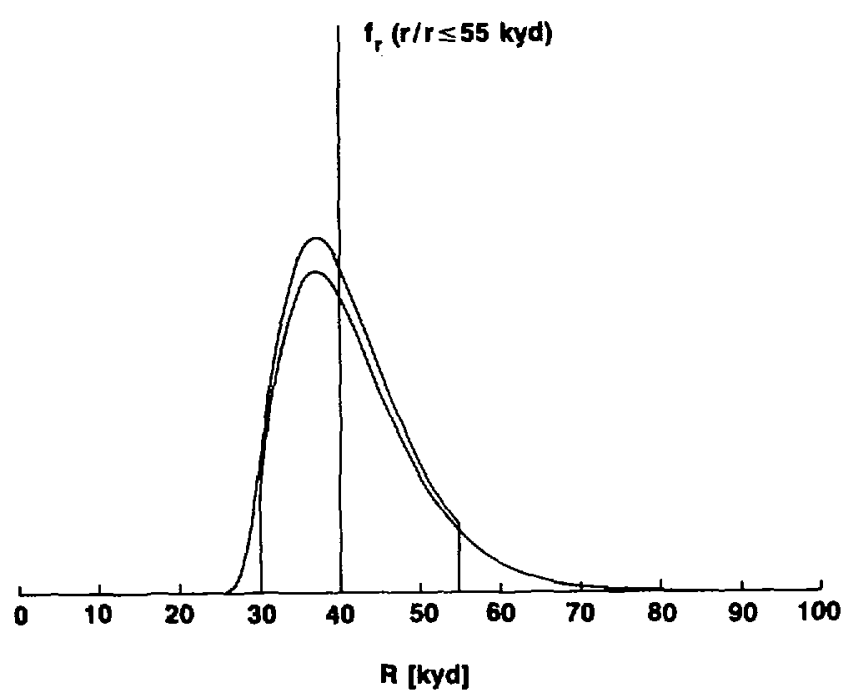

Fig. 6. Conditional range density function-one gate.

\section{Statistical Results}

The conditional probability functions as well as the firstand second-order moments for both the time-delay measurement errors and the corresponding estimated range were developed in the preceding sections. In this section the range 


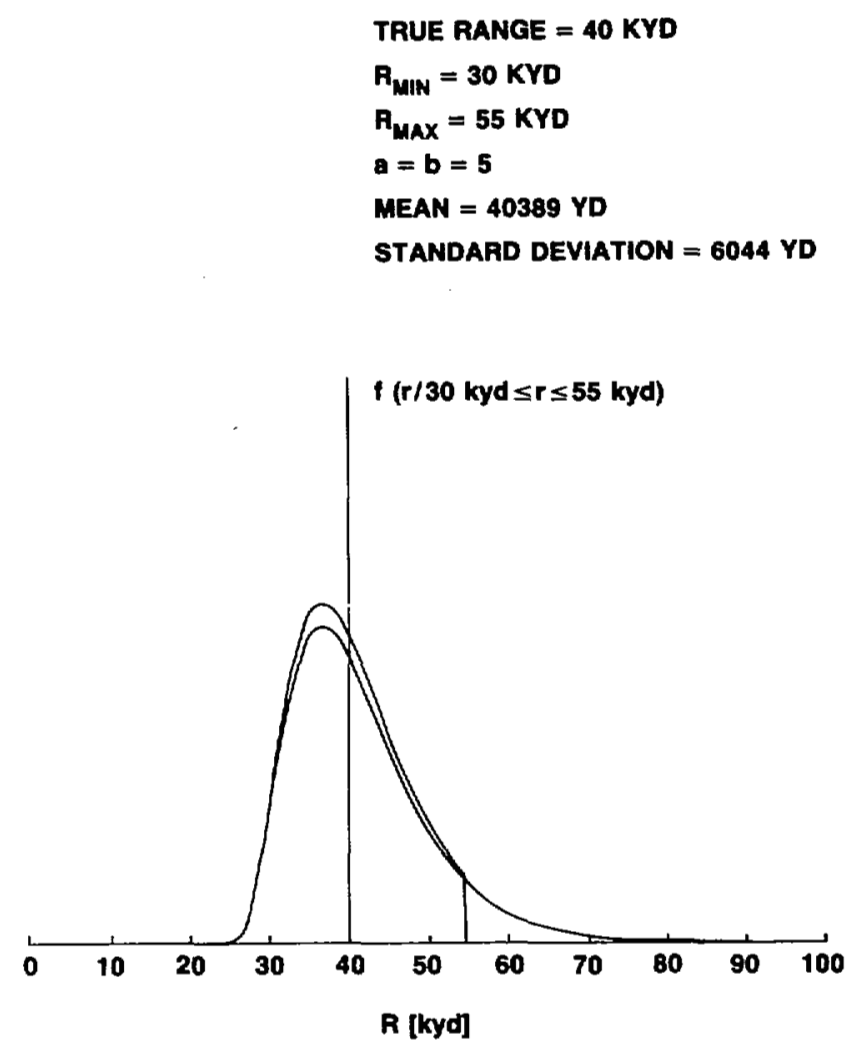

Fig. 7. Conditional range density function-two gates.

statistics are compared against the statistics obtained from previous ungated analyses [7] which limited the end points of the beta density function. The mean $m_{r}$ and standard deviation $\sigma_{r}$ for both the gated and nongated range statistics are plotted against the measurement error standard deviation $\sigma_{\epsilon}$ in Fig. 8 for a true range of $40 \mathrm{kyd}$. When no gates are used, $m_{r}$ and $\sigma_{r}$ approach infinity as $\sigma_{\epsilon}$ increases. When the measurement error is gated by either one or two gates, $m_{r}$ and $\sigma_{r}$ tend to level out or decrease. For small values of $\sigma_{\epsilon}$, the gated and nongated range statistics are equivalent. The values begin to separate at $\sigma_{\epsilon}=0.01 \mathrm{~ms}$. As $\sigma_{\epsilon}$ increases the gated statistics reach a maximum value and then decrease. This maximum value is a function of gate placement as well as whether one or more gates are used. Fig. 8 also demonstrates that unless the gates are set properly, which implies a priori information about the true range, range estimates are reliable for only small values of $\sigma_{\epsilon}$.

The plots in Fig. 8 were generated from a time-delay error density function with the positive end point less than the maximum allowable value $\left(A_{1} \leq E / c\right)$; therefore, the use of the conditional density function was not necessary. If $A_{1}>E$ / $c$ then gating is essential to ensure that infinite ranges or negative ranges do not occur. Fig. 9 contains plots for $m_{r}$ and $\sigma_{r}$ when $A_{1}>E / C$ for true range values of 5 to $30 \mathrm{kyd}$. A single gate is placed on the time-delay measurement so that the upper range estimate limit is less than twice the true range value. Both $m_{r}$ and $\sigma_{r}$ reach a maximum value and then decrease as $\sigma_{\epsilon}$ increases. These plots are very similar to those obtained in [4] when a Gaussian distribution was employed to model the measurement errors.
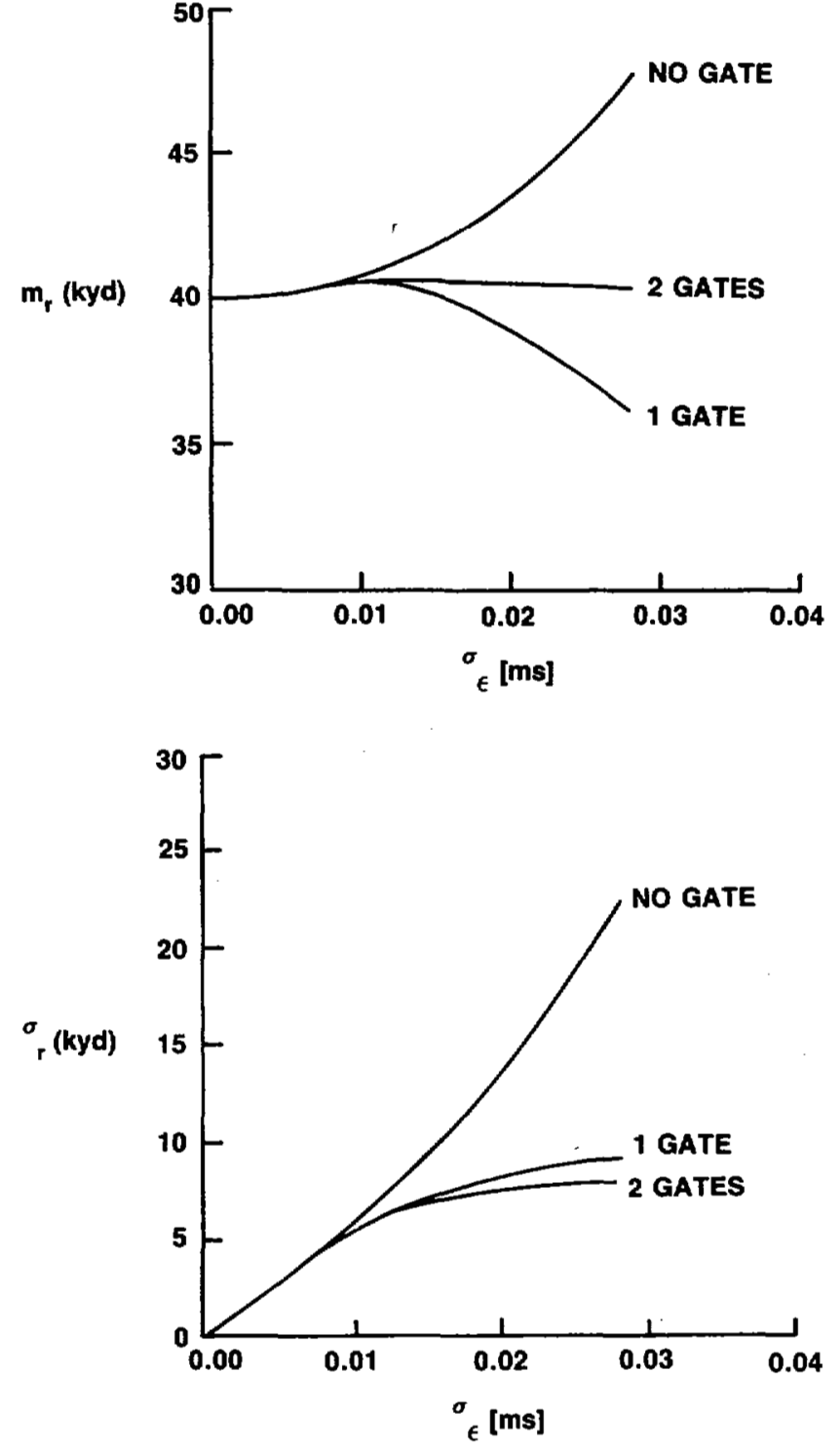

Fig. 8. Range statistics for zero, one, and two range gates.

\section{Comparison of the Range Statistics for Beta and Gaussian Time-Delay Errors}

In the previous section, an analytical formulation of the estimated range statistics was developed when a conditional beta density was employed to model time-delay measurement errors. This conditional beta density function enables the standard deviation for the measurement errors to be extended so that a comparison of estimated range statistics can be made between the beta distribution and the gated Gaussian distribution.

The estimated range statistics for gated Gaussian time-delay measurement errors were obtained through computer simulation since an analytical solution was not available. MonteCarlo runs were made to determine the mean and variance of the estimated range for various Gaussian time-delay standard deviations. Fig. 10(a) and (b) shows estimated range statistics for both the beta and Gaussian time-delay measurement errors. The true range is $30 \mathrm{kyds}$ and a range gate is placed at twice the true range $\left(R_{\max }=60 \mathrm{kyd}\right)$. Both the beta and Gaussian 

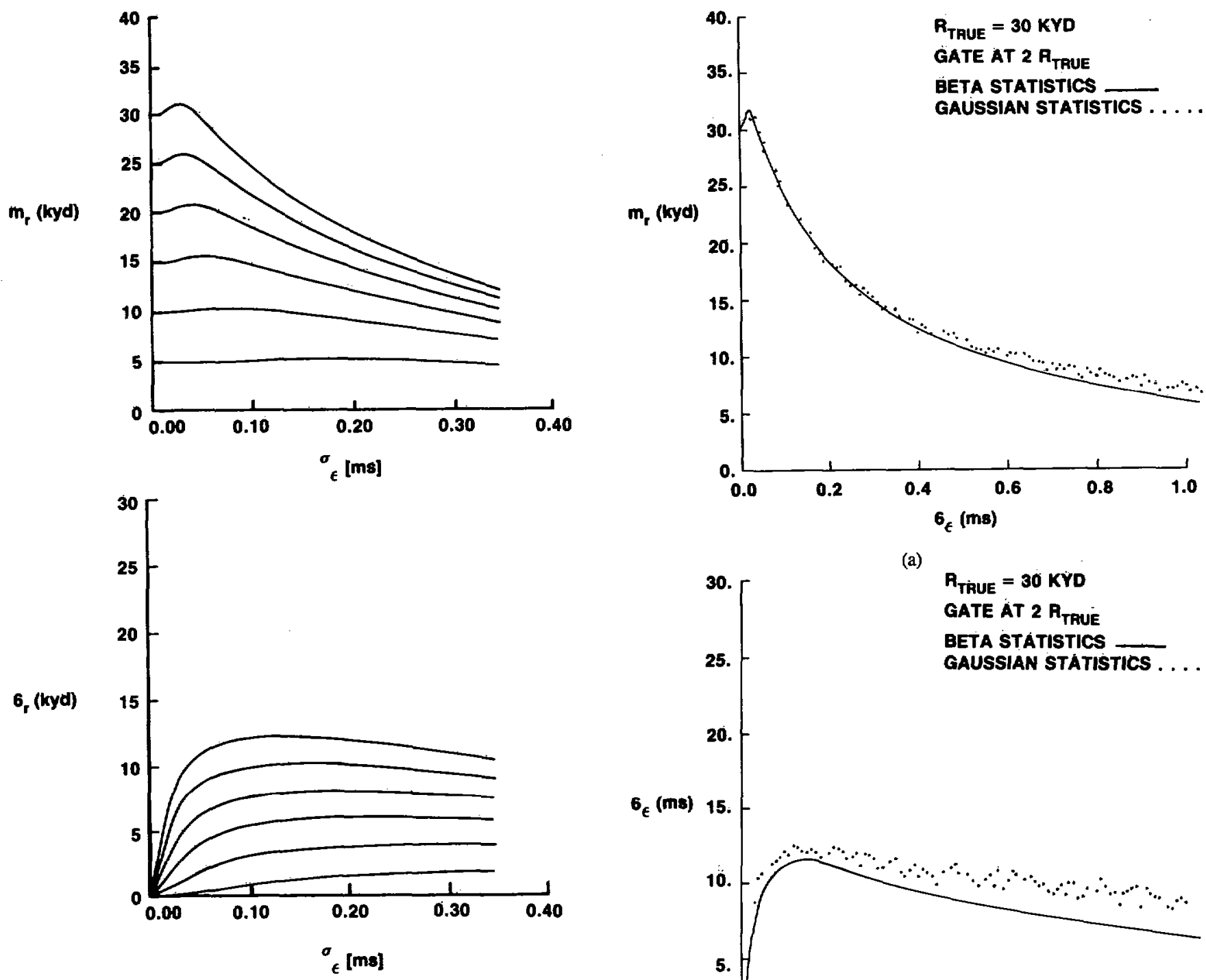

Fig. 9. Range statistics for rang gate at $2 R_{T}$.

range statistic plots have the same shape; that is, the estimated range statistics begin to increase as $\sigma_{\epsilon}$ increases until the range gate takes effect, which then causes the range statistics to decrease in value. The statistics are almost identical for small $\sigma_{\epsilon}$ values, but as $\sigma_{\epsilon}$ increases, the beta and Gaussian range statistics begin to deviate. The characteristics of the standard deviation plot (Fig. 10(b)) are similar to those of the mean range plot (Fig. 10(a)), except the difference between the Gaussian and beta range statistics is larger as $\sigma_{\mathrm{f}}$ increases. More insight into similarities and differences of the range statistics can be gained by examining the density function in Fig. 4(a)-(c). This figure reveals how statistics for both the truncated beta and Gaussian density functions change as a result of the initial $\sigma$ of the measurement errors. For small values of $\sigma$, Fig. 4(a), shows that the two densities are very nearly identical. As $\sigma$ increases (Fig. 4(b) and (c)), the two density functions show increasing differences, but still have similar features.

\section{CONCLUSIONS}

Analytical expressions for the estimated range density function, its mean, and standard deviation were formulated by

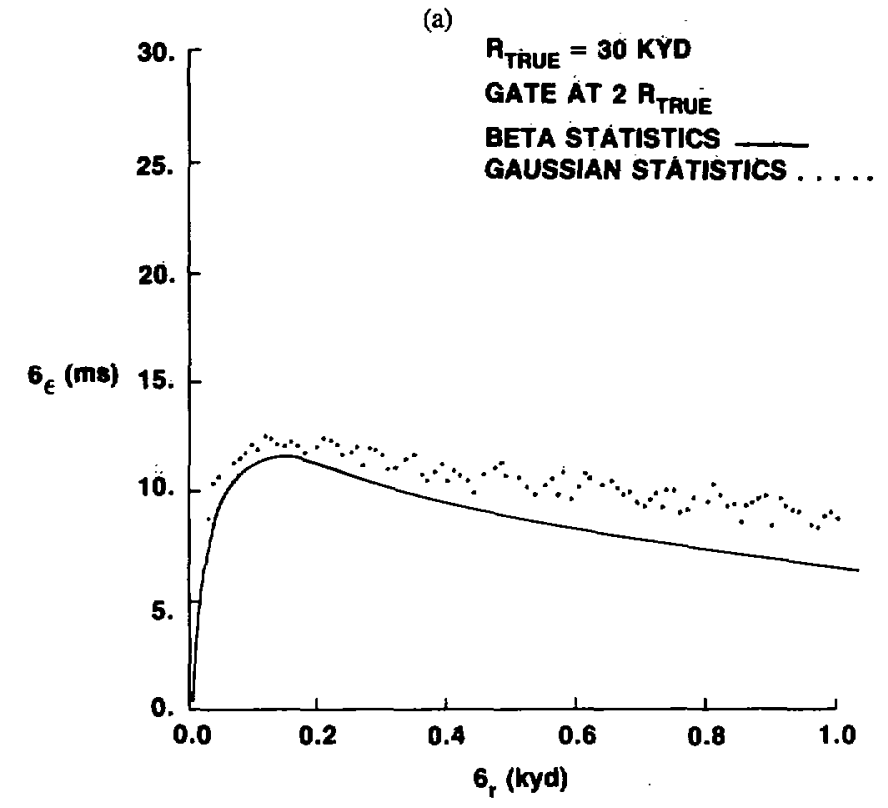

(b)

Fig. 10. (a) Mean range for beta and Gaussian measurement errors: (b) Range standard deviation for beta and Gaussian measurement errors

employing a beta-type probability function to model tímedelay measurement errors. These analytical expressions are essential for developing effective search patterns, since contour regions may then be generated and areas of high source location probability identifled. This paper has demonstrated the validity and effectiveness of beta distributions by comparing the resulting analytical statistics with the simulated statistics obtained when conventional Gaussian distributions were used to model time-delay measurement errors. These results show that there is very little difference in the range statistics, and, in fact, for small measurement errors, they are nearly identical.

In summary, the flexibility and mathematical tractability exhibited by beta density functions render them attractive candidates for modeling time-delay measurement errors. By providing added flexibility in parameter selection, biased and/ 
or asymmetric measurement data can be modeled. Skewed error distributions may also be described by simply varying the shaping parameters. Similarly, the modeling of measurement bias is readily achieved through proper selection of end points and/or shaping parameters.

\section{ACKNOWLEDGMENT}

The authors gratefully acknowledge V. Aidala and J. Baylog for their contributions to this work, and D. Champion for her patience in preparation of this paper.

\section{REFERENCES}

[1] G. C. Carter, "Variance bounds for passively locating an acoustic source with symmetric line array," J. Acoust. Soc. Amer., vol. 62 , pp. 922-926, 1977.

[2] G. C. Carter, "A brief description of the fundamental difficulties in passive ranging," IEEE J. Ocean. Eng., vol. OE-3, pp. 65-66, 1978.

[3] E. J. Hilliard, Jr. and R. F. Pinkos, "An analysis of triangulation ranging using beta density angular errors," J. Acoust. Soc. Amer., vol. 65 , no. 5 , pp. $1218-1228,1979$

[4] J. C. Hassab and R. E. Boucher, "Passive ranging estimation from an array of sensors," J. Sound Vibration, vol. 67, no. 2, pp. 289-292, 1979.

[5] L. C. Ludeman, "Bias and variance of a sound ranging estimator," Dept. Elec. and Comput. Eng., New Mexico State Univ., Las Cruces, NM, 1979.

[6] E. J. Hilliard, Jr. and J. J. Perruzzi, "Time-delay triangulation ranging using beta density time-delay errors," J. Acoust. Soc. Amer., vol. 72, no. 6, pp. 1831-1837, Dec. 1982.

[7] J. J. Perruzzi and E. J. Hilliard, Jr., "Modeling time-delay measurement errors using a generalized beta density function," J. Acoust. Soc. Amer., vol. 75, no. 1, pp. 197-201, Jan. 1984.

[8] J. P. Ianniello, "Time delay estimation via cross-correlation in the presence of large estimation errors," IEEE Trans. Acoust., Speech, Signal Processing, vol. ASSP-30, pp. 998-1003, Dec. 1982.

[9] A. J. Weiss and E. Weinstein, "Fundamental limitations in passive time delay estimation-Part I: Narrow-band systems," IEEE Trans. Acoust., Speech, Signal Processing, vol. ASSP-31, pp. 472-486, Apr. 1983.

[10] J. P. Ianniello, "Lower bounds on worst case probability of large error for two channel time delay estimation," IEEE Trans. Acoust., Speech, Signal Processing, vol. ASSP-33, pp. 1102-1110, Oct. 1985.

[11] A. Papoulis, Probability, Random Variables and Stochastic Processes. New York: McGraw-Hill, 1965.

[12] I. S. Gradshteyn and I. M. Ryzhik, Table of Integrals, Series, and Products, 4th ed. New York: Academic, 1965.

[13] H. Exton, Handbook of Hypergeometric Integrals Theory, Applications, Tables, Computer Programs. New York: Wiley, 1978.

[14] W. C. Griffin, Transform Techniques for Probability Modeling. New York: Academic, 1975.

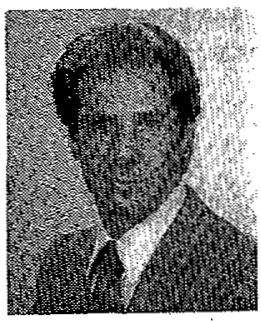

Joseph J. Perruzzi (S'78-M'78) received the B.S. and M.S. degrees in electrical engineering from Southeastern Massachusetts University, North Dartmouth, in 1972 and 1977, respectively.

Since 1973, he has been employed by the Naval Underwater Systems Center in Newport, RI, and currently holds the position of Senior Systems Analyst. His principal activity is involved in the development and implementation of adyanced guidance and control concepts for underwater weapons. Current research interests include stochastic modeling of effectiveness measures and optimization of weapon performance.

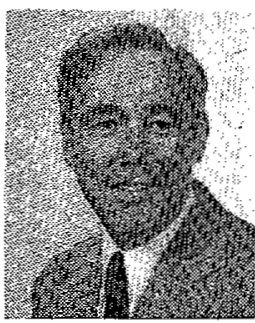

Edward J, Hilliard, Jr. (S'47-A'48-M'64) was born in Pittsfield, MA, on May 31, 1926. He received the $B . E$. degree in electrical engineering from Yale University, New Haven, CT, in 1948. He has done graduate work at both the University of Virginia, Charlottesville, and the University of Rhode Island, Kingston.

From 1948 until 1953 he was employed as an Acronautical Research Scientist at the Langley Laboratory of NASA where he was involved in the development and design of radio telemetry systems. In 1953 he moved to the Naval Torpedo Station, now the U.S. Naval Underwater Systems Center, Newport, RI. From that date to the present he has done research, development, and design work in various sectors of the underwater missile field. In the course of that work he has published over 55 reports, papers, and articles, some of which include analysis of communication with underwater vehicles by radio and wire, the geometry of evading targets, and the statistics of triangulation ranging using non-Gaussian error densities. He holds two U.S. patents for signal processing circuits.

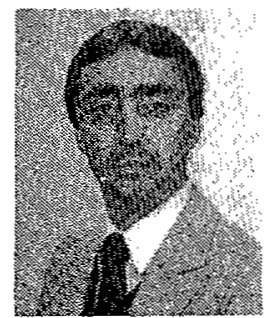

David V. Pereira received the B.S. degree in electrical engineering in 1971 and the M.S. degree in physics in 1976 both from Southeastern Massachusetts University, North Dartmouth. He has been accepted as a Ph.D. degree candidate in the applied mathematics program at the University of Rhode Island, Kingston.

Since 1980 he has been employed by the Naval Underwater Systems Center, Newport, RI, in the Combat Control Systems Department. Since joining NUSC he has served in various engineering capacities involving software, hardware, and systems development. He is currently working for the Weapon Setting and Control Branch and is involved in the research and development of advanced weapon concepts. 